\title{
The efficacy of synovectomy for total knee arthroplasty: a meta-analysis
}

\author{
Zi-gin Zhao, Jin Xu', Rui-lin Wang and Li-na Xu
}

\begin{abstract}
Background: Many studies have proposed synovectomy during total knee arthroplasty (TKA) to reduce pain after TKA. The aim of this study was to assess the outcomes of synovectomy for treating of TKA through a meta-analysis.

Methods: Relevant clinical studies on synovectomy and without synovectomy were retrieved through searching the databases PubMed, Embase, Web of Science, and the Cochrane Central Register of Controlled Trials up to January 2018. Studies that investigated the comparison of pain scores, total blood loss, range of motion, functional Knee Society Scores (KSSs), clinical KSSs, and operating time and provided sufficient data of interest were included in this meta-analysis. Stata 12.0 was used for meta-analysis.
\end{abstract}

Results: Ten randomized controlled trials (RCTs) were finally included in this meta-analysis. Final results indicated that there was no significant difference between the pain scores, range of motion, functional Knee Society Scores (KSSs), and clinical KSSs $(P>0.05)$. However, synovectomy was associated with an increase of the total blood loss compared to patients without synovectomy (weighted mean difference $(\mathrm{WMD})=116.71,95 \%$ confidence interval $(\mathrm{Cl}) 78.63,154$. $79, P=0.000$ ). Pooled results indicated that synovectomy was associated with an increase of the operating time (WMD $=15.44,95 \% \mathrm{Cl} 2.67,28.21, P=0.018)$.

Conclusions: Current evidence indicates that synovectomy has no effects on the final clinical outcomes for patients undergoing TKA. It will increase the total blood loss and the operating time during TKA.

Keywords: Synovectomy, Total knee arthroplasty, Meta-analysis

\section{Background}

Synovial proliferation is a common intraoperative finding in patients with osteoarthritis (OA) or rheumatoid arthritis (RA) of the knee [1]. Synovectomy when in total knee arthroplasty (TKA) for primary OA, excising the inflamed or proliferated synovial membrane, reduces pain and improves joint function and is beneficial for patients with RA and other inflammatory disease [2]. Although many inflammatory cytokines have been isolated from the knee and the blood in patients with moderate to severe primary osteoarthritis, [3] the benefit of synovectomy as a sole procedure in OA remains unclear.

Most studies have reported favorable results following synovectomy combined with other specific surgical procedures [4]. Synovectomy undertaken during total knee arthroplasty (TKA) depends on the surgeon's preference

\footnotetext{
* Correspondence: xuajin511@163.com

Department of Pathology, Tianjin Hospital, No. 406 Jiefang South Road, Hexi

District, Tianjin 300211, People's Republic of China
}

[5]. Krackow et al. [6] recommended that as little synovium as possible should be removed. Synovitis has been reported as one of several contributing factors in unsatisfactory results after TKA [7]. This raises the question as to whether intraoperative synovectomy during TKR would be advantageous in decreasing postoperative inflammation of the knee.

A previous meta-analysis compared synovectomy to without synovectomy for clinical outcomes in patients who underwent TKA [8]. However, the disadvantages were as follows: (1) only three studies were included and thus will increase the type I error probability; (2) operating time was not compared; and (3) additionally, more evidence is emerging, and it is necessary to re-evaluate the efficacy of synovectomy for clinical outcomes after TKA. This meta-analysis aimed to evaluate whether synovectomy can decrease pain intensity and increase clinical outcomes after TKA. 
Table 1 The general characteristics of the included studies. 1 pain scores, 2 total blood loss, 3 range of motion, 4 clinical KSSs, 5 functional KSSs, 6 operating time NS not stated, RCTs randomized controlled trials

\begin{tabular}{lllllllll}
\hline Author & Mean age & Male patients (\%) & Cases & Intervention & Control & Outcomes & Follow-up & Study \\
\hline Dong 2016 [11] & 65 & 43.5 & 60 & Synovectomy & Synovium-retaining & $3,4,5,6$ & 12 months & RCTs \\
Hua 2015 [12] & 70 & 55.6 & 187 & Synovectomy & Synovium-retaining & $1,2,3,4,5,6$ & At discharge & RCTs \\
Kilicarslan 2011 [13] & 68 & 34.7 & 100 & Synovectomy & Synovium-retaining & $1,2,3,4,5,6$ & 6 months & RCTs \\
Li 2014 [14] & 67 & 44.1 & 90 & Synovectomy & Synovium-retaining & $3,4,5,6$ & NS & RCTs \\
Ning 2014 [15] & 67 & 52.4 & 374 & Synovectomy & Synovium-retaining & $1,2,3,4,5,6$ & NS & RCTs \\
Shaoning 2014 [16] & 58 & 48.2 & 187 & Synovectomy & Synovium-retaining & $1,2,3,4$, & NS & RCTs \\
Tanavalee 2011 [17] & 70 & 41.6 & 67 & Synovectomy & Synovium-retaining & $1,2,3,4,5,6$ & 3 months & RCTs \\
Yang 2016 [18] & 67 & 45.4 & 118 & Synovectomy & Synovium-retaining & $1,2,3,4,5,6$ & NS & RCTs \\
Zhaoning 2013 [5] & 65 & 38.7 & 187 & Synovectomy & Synovium-retaining & $1,2,3,4,5,6$ & 12 months & RCTs \\
Hu 2017 [19] & 70 & 42.5 & 105 & Synovectomy & Synovium-retaining & $1,2,3,4$ & 3 months & RCTs \\
\hline
\end{tabular}

\section{Methods}

\section{Literature search}

The electronic databases PubMed, Embase, and Cochrane Central Register of Controlled Trials were searched for all articles on synovectomy and without synovectomy for treating OA patients. The following were the search terms: (synovectomy OR) AND (TKA OR TKR OR total knee arthroplasty OR total knee replacement OR "Arthroplasty, Replacement, Knee"[Mesh]) where the search date was January 2018. In addition, a manual search of the bibliographies of the identified articles was performed to elucidate potentially relevant studies. The reliability of the study selection was determined by Cohen's kappa test, and the acceptable threshold value was set at $0.61[9,10]$.

\section{Inclusion and exclusion criteria}

Abstracts of all citations and retrieved studies were reviewed. Studies meeting the following criteria were included: (1) patients prepared for TKA, (2) randomized controlled trial (RCTs), (3) intervention was synovectomy and control was without synovectomy for TKA, and (4) outcomes including pain scores, total blood loss, range of motion, functional Knee Society Scores (KSSs), clinical KSSs, and operating time.

Studies were excluded if one of the following existed: providing undefined sample and control source, nontherapeutic clinical studies, animal experiments, nonoriginal studies, and undefined grouping.

\section{Quality assessment}

The methodological quality of all included trials was independently assessed by two reviewers using the Cochrane Handbook for Systematic Reviews of Interventions, version 5.1 .0 (http://handbook.cochrane.org/). A total of seven items (random sequence generation, allocation concealment, blinding to the participant and personnel, blinding to the outcome assessment, incomplete outcome, selective reporting, and other bias) were measured. Each of the items was measured as "low risk of bias," "unclear risk of bias," and "high risk of bias." The risk of bias summary and risk of bias graph were obtained using Review Manager 5.3.0 software (The Nordic Cochrane Centre, The Cochrane Collaboration, Copenhagen, Denmark).

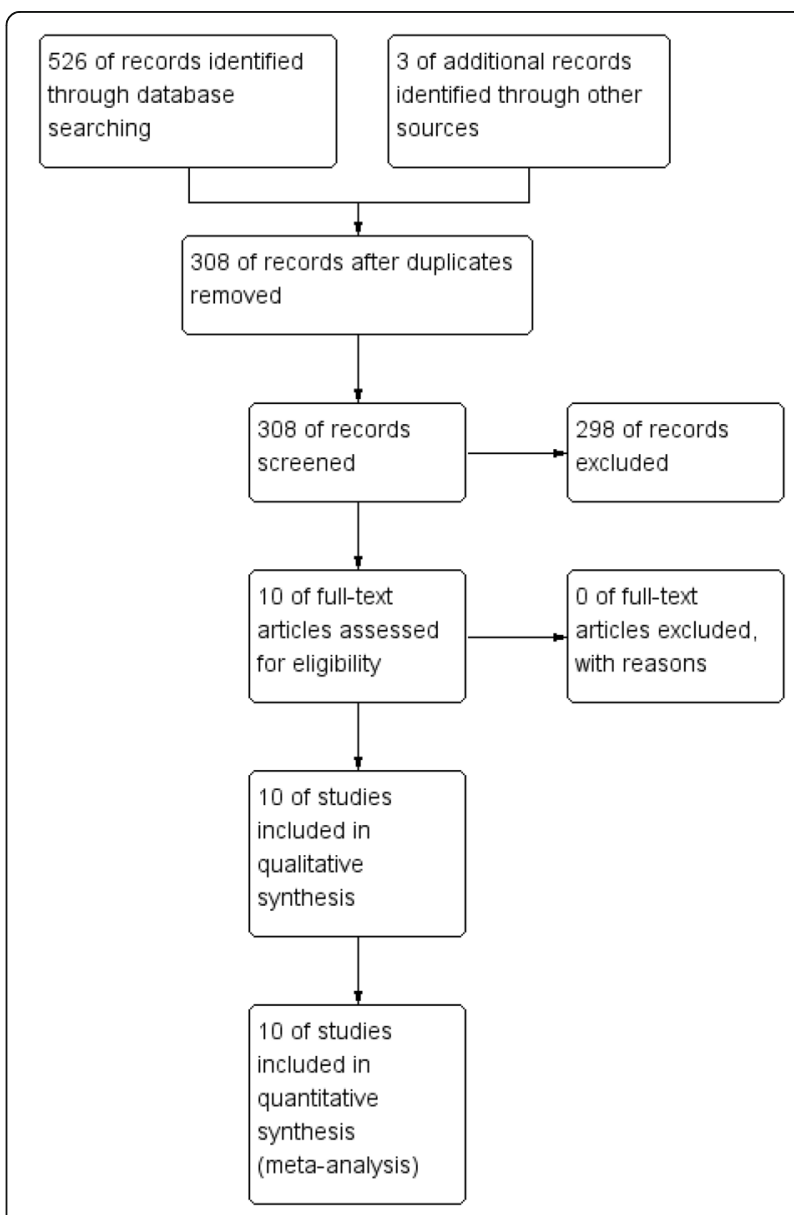

Fig. 1 Flowchart of study search and inclusion criteria 


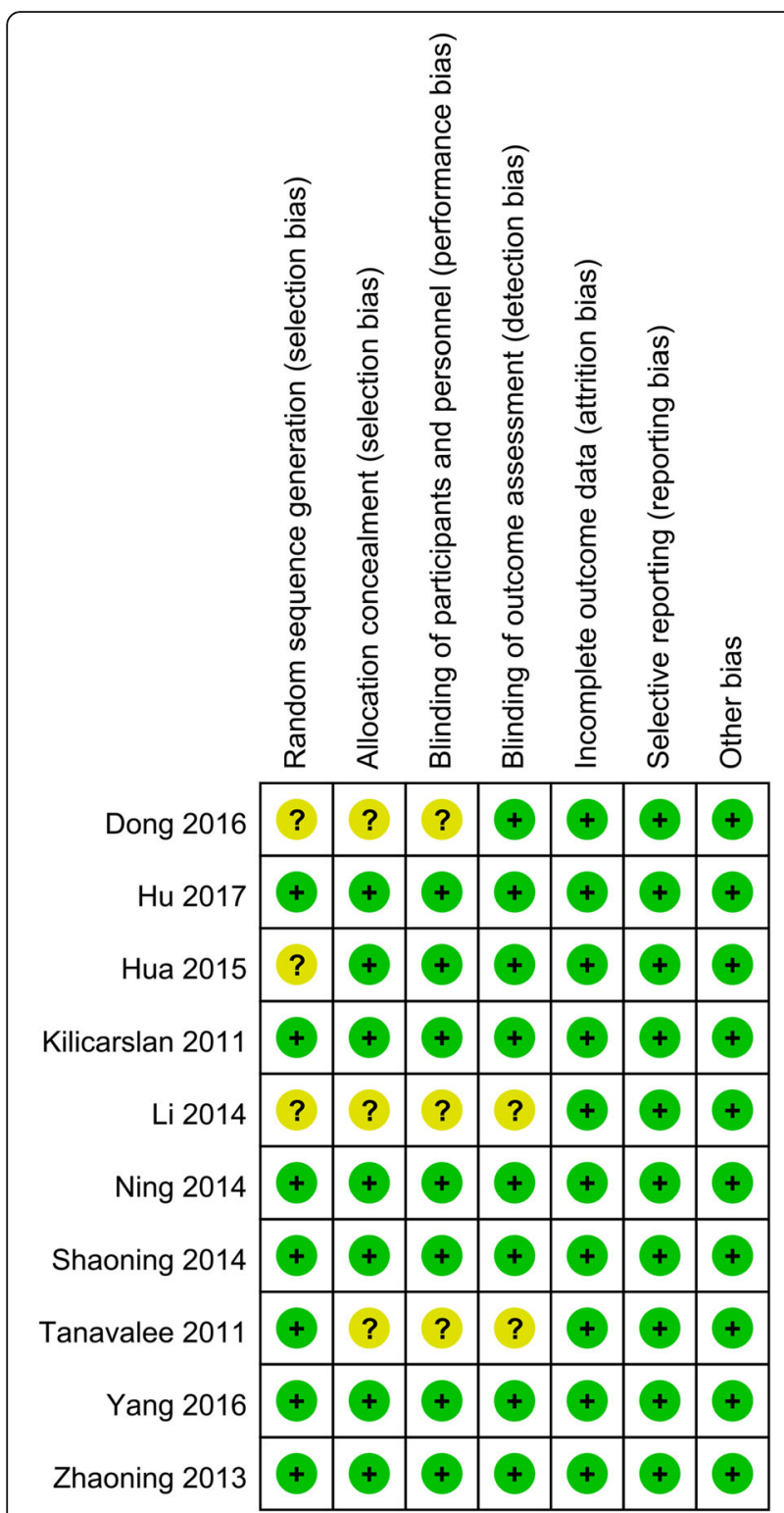

Fig. 2 Risk-of-bias summary of included randomized controlled trials. + , no bias; -, bias; ?, bias unknown

\section{Data extraction}

A specific extraction was performed to collect the following data from the included trials: patients' general characteristics, intervention, control group, outcomes, study, and follow-up. Two reviewers independently extracted the relevant data from the published articles. Outcomes such as pain scores, total blood loss, range of motion, functional KSSs, clinical KSSs, and operating time were abstracted and recorded on a form (Table 1). In studies in which data were incomplete or unclear, attempts were made to contact investigators for clarification. All data were extracted by two independent reviewers, and disagreements were resolved by discussion.

\section{Statistical analysis}

The statistical analysis was conducted using Stata 12.0 software. Continuous outcomes (total blood loss, range of motion, functional KSSs, clinical KSSs, and operating time) were expressed as weighted mean differences (WMD) and 95\% confidence interval (CI). Pain scores were expressed as standard mean difference (SMD) and 95\% CI as they use different methods to assess the pain intensity. $P<0.05$ was considered statistically significant. Heterogeneity was assessed with the $\chi^{2}$-based $\mathrm{Q}$ testing. If there was significant heterogeneity $(P<0.1)$, we selected a random-effects model to pool the data. If not, a fixed effects model was used. Publication bias was tested using funnel plots and Begg's test $(P>0.05$ was identified as no publication bias).

\section{Results}

Literature characteristics

Figure 1 presents the selection of the included studies. In the initial search, we identified 526 potentially relevant studies and 3 additional records through other sources. Among the 529 articles, 221 duplicates were removed by Endnote Software (Version X7, Thompson Reuters, CA, USA). According to the inclusion criteria, 298 studies were excluded after reading the titles and abstracts. Finally, we included 10 clinical trials in the meta-analysis [5, 11-19].

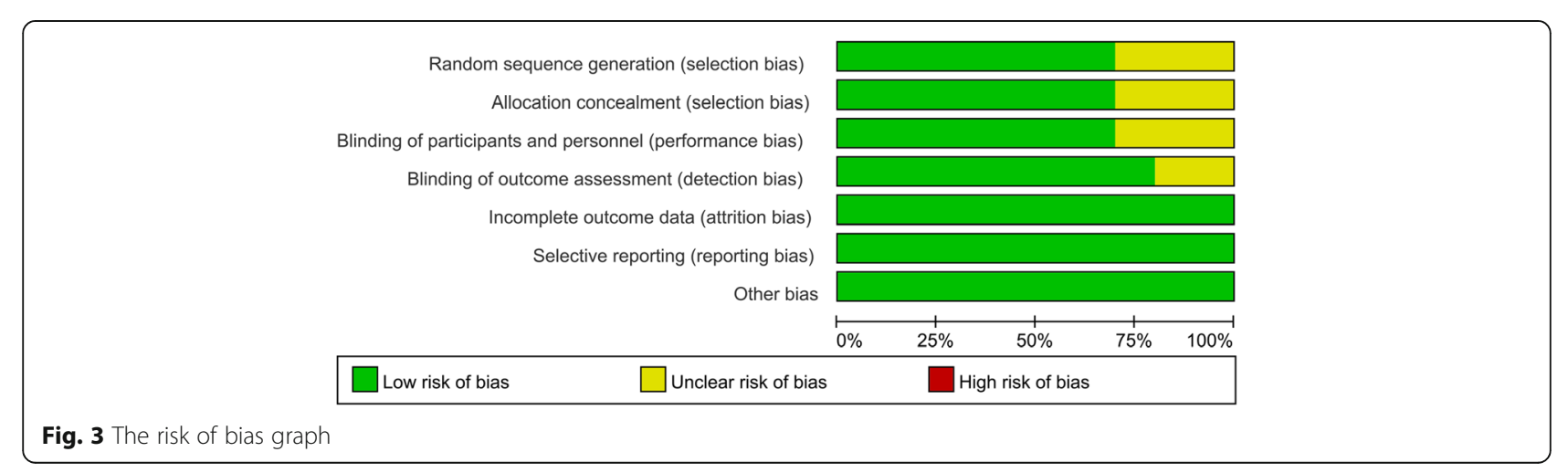




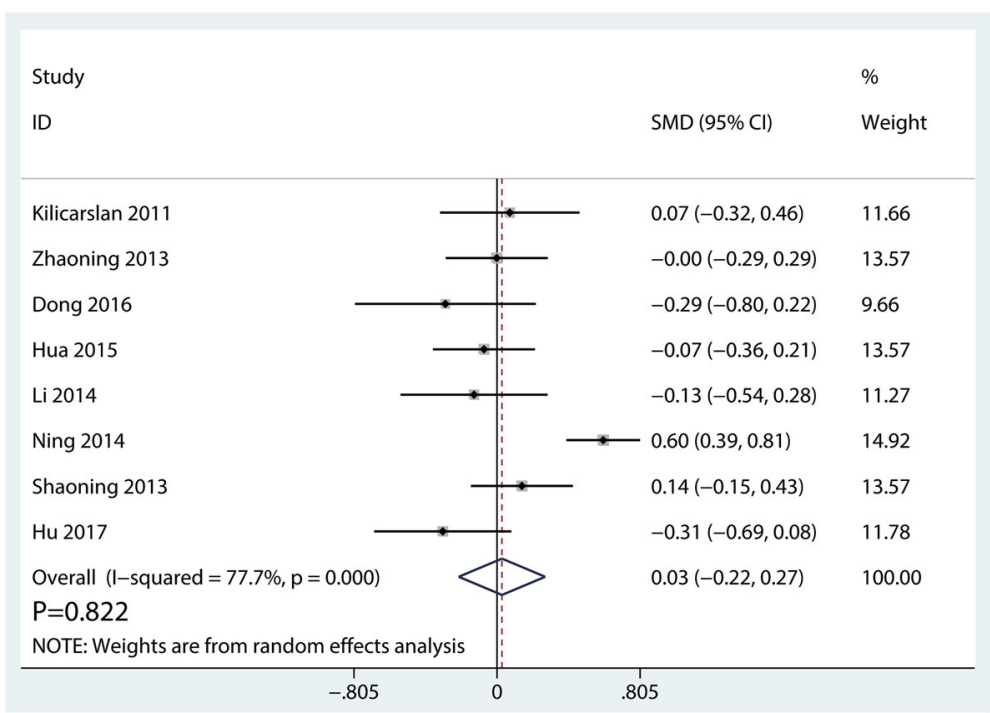

Fig. 4 Forest plots of the included studies comparing the pain scores

\section{Quality assessment}

The kappa value between the two reviewers was 0.803 , which indicated that there was good agreement between the two reviewers. The quality assessment of the included studies is summarized in Figs. 2 and 3. Seven studies refer to the random sequence generation and the rest three studies did not state the random sequence generation and identify as unclear risk of bias. One of the seven studies refers to the allocation concealment and identifies as low risk of bias. Other risks of bias are all with unclear risk of bias. Two studies did not refer to the blinding of the participants and outcome assessment. Other biases are all with low risk of bias.

\section{Results of meta-analysis Pain scores}

We obtained usable data on pain scores from eight trials including 1290 knees. As depicted in Fig. 4, there was significant heterogeneity $\left(I^{2}=77.7 \%, P=0.000\right)$, and thus, we used a random-effects model. And the pooled results indicated that there was no significant difference between synovectomy group and control group in terms

\begin{tabular}{|c|c|c|c|}
\hline \multirow{2}{*}{\multicolumn{2}{|c|}{ Study }} & \multirow[b]{2}{*}{ WMD (95\% Cl) } & \multirow{2}{*}{$\begin{array}{l}\% \\
\text { Weight }\end{array}$} \\
\hline & & & \\
\hline Kilicarslan 2011 & $\rightarrow$ & $107.00(33.98,180.02)$ & 14.71 \\
\hline Zhaoning 2013 & $\rightarrow$ & $90.00(8.66,171.34)$ & 13.01 \\
\hline Dong 2016 & - & $428.00(216.21,639.79)$ & 2.94 \\
\hline Hua 2015 & $\rightarrow$ & $90.00(8.82,171.18)$ & 13.04 \\
\hline Li 2014 & - & $180.00(82.35,277.65)$ & 10.31 \\
\hline Ning 2014 & $\rightarrow$ & $120.00(61.05,178.95)$ & 18.12 \\
\hline Shaoning 2013 & $\rightarrow$ & $90.00(15.44,164.56)$ & 14.38 \\
\hline Hu 2017 & $\rightarrow$ & $86.80(7.85,165.75)$ & 13.48 \\
\hline $\begin{array}{l}\text { Overall (I-squared }=40.6 \%, p=0.108) \\
P=0.000 \\
\text { NOTE: Weights are from random effects analysis }\end{array}$ & $\hat{3}$ & $116.71(78.63,154.79)$ & 100.00 \\
\hline-640 & 0 & 40 & \\
\hline
\end{tabular}




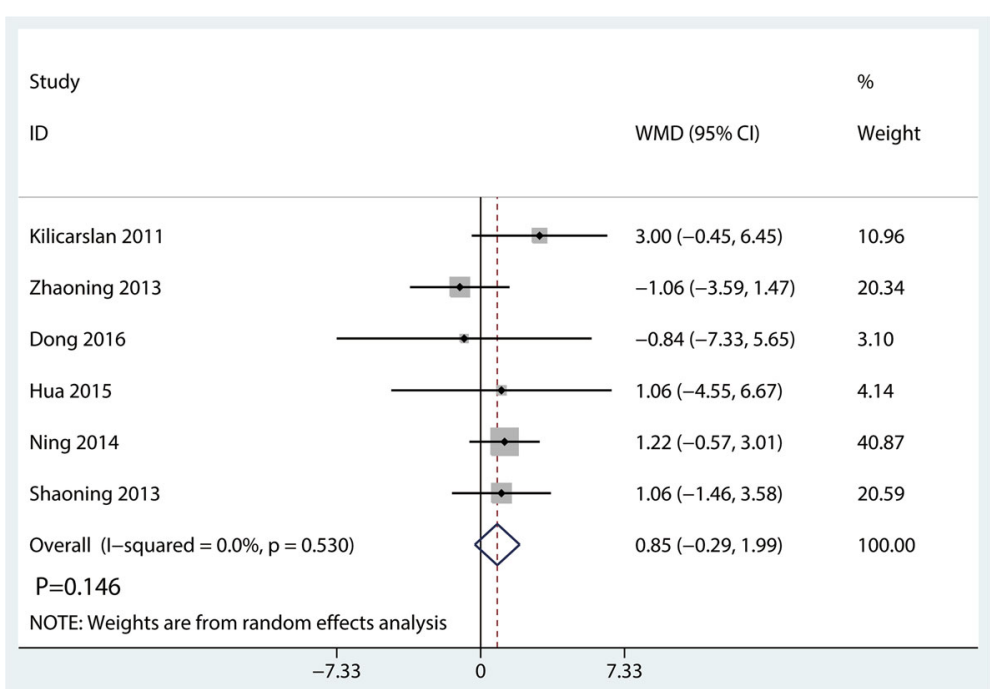

Fig. 6 Forest plots of the included studies comparing the range of motion

of the postoperative pain scores $(\mathrm{SMD}=0.03,95 \% \mathrm{CI}-$ $0.22,0.27, P=0.822$ ).

\section{Total blood loss}

Total blood loss was reported in eight studies, and the pooled results indicated that the synovectomy group was associated with an increase of the total blood loss (WMD = 116.71, 95\% CI 78.63, 154.79, $P=$ 0.000, Fig. 5). A random-effects model was used because statistical heterogeneity was found between the studies $\left(I^{2}=40.6 \%, P=0.108\right)$.

\section{Range of motion}

Range of motion was mentioned in six studies; the pooled results showed no significant heterogeneity $\left(I^{2}=\right.$ $0.0 \%, P=0.530)$. And the pooled results indicated that there was no significant difference between the synovectomy group and control group in terms of the range of motion (WMD $=0.85,95 \%$ CI $-0.29,1.99, P=0.146$, Fig. 6).

\section{Clinical KSS}

Clinical KSSs were reported in seven studies, and the pooled results indicated that there was no significant difference between the synovectomy group and control group in

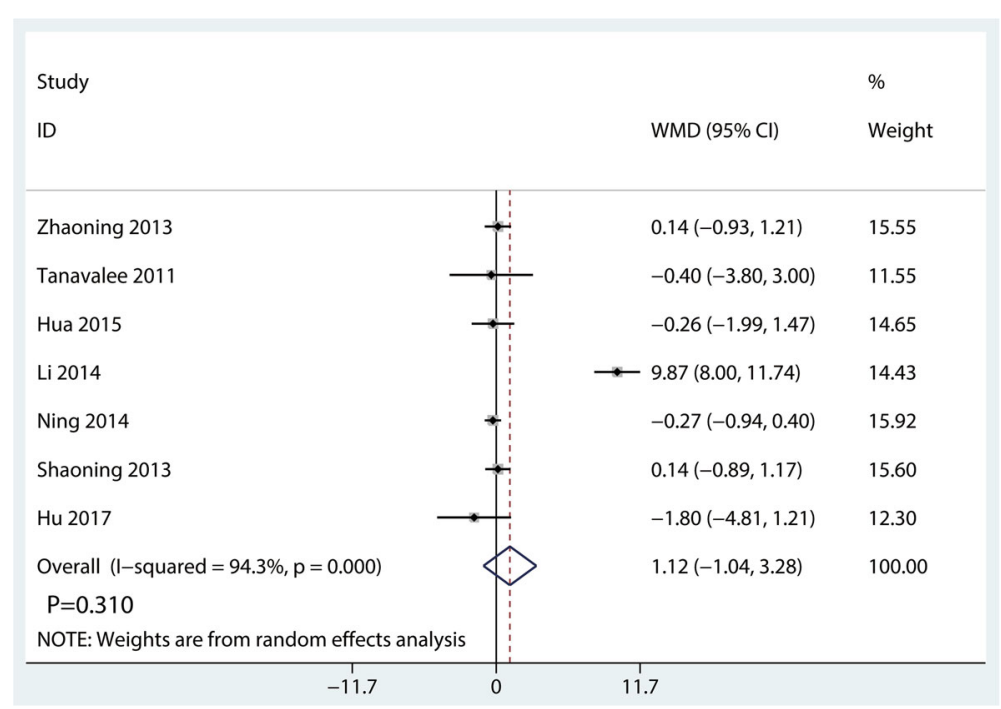

Fig. 7 Forest plots of the included studies comparing the clinical KSS 


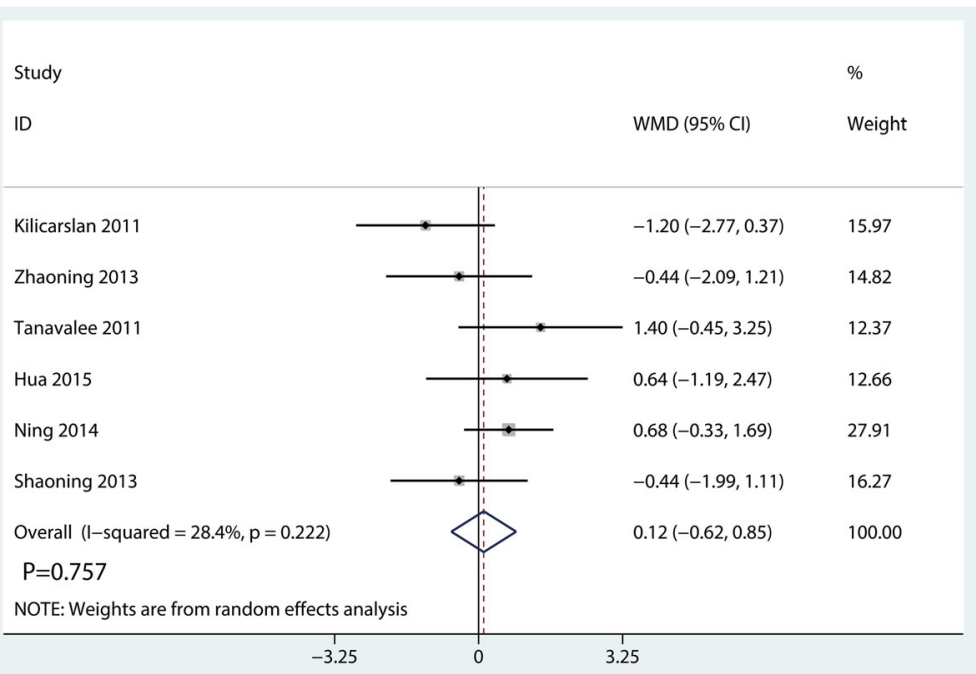

Fig. 8 Forest plots of the included studies comparing the functional KSS

terms of the clinical KSS (WMD $=1.12,95 \%$ CI -1.04 , 3.28, $P=0.310$, Fig. 7 ).

\section{Functional KSS}

Functional KSSs were reported in six studies, and the pooled results indicated that there was no significant difference between the synovectomy group and control group in terms of the functional KSS (WMD $=0.12,95 \% \mathrm{CI}-0.62$, $0.85, P=0.757$, Fig. 8).

\section{Operating time}

Operating time was reported in three studies, and the pooled results indicated that synovectomy was associated with an increase of the operating time (WMD $=15.44$, 95\% CI 2.67, 28.21, $P=0.018$, Fig. 9).

\section{Funnel plot and sensitivity analysis}

Funnel plot and Begg's test of pain scores are seen in Figs. 10 and 11, respectively. Results show that there was a potential publication bias for the pain scores $(P=0.012)$. Sensitivity analysis shows that after excluding the studies one by one, the entire results were not changed (Fig. 12).

\section{Discussion}

Current meta-analysis indicated that, compared with control group, synovectomy in conjunction with TKA for primary OA provides no clinical benefits. Results have shown

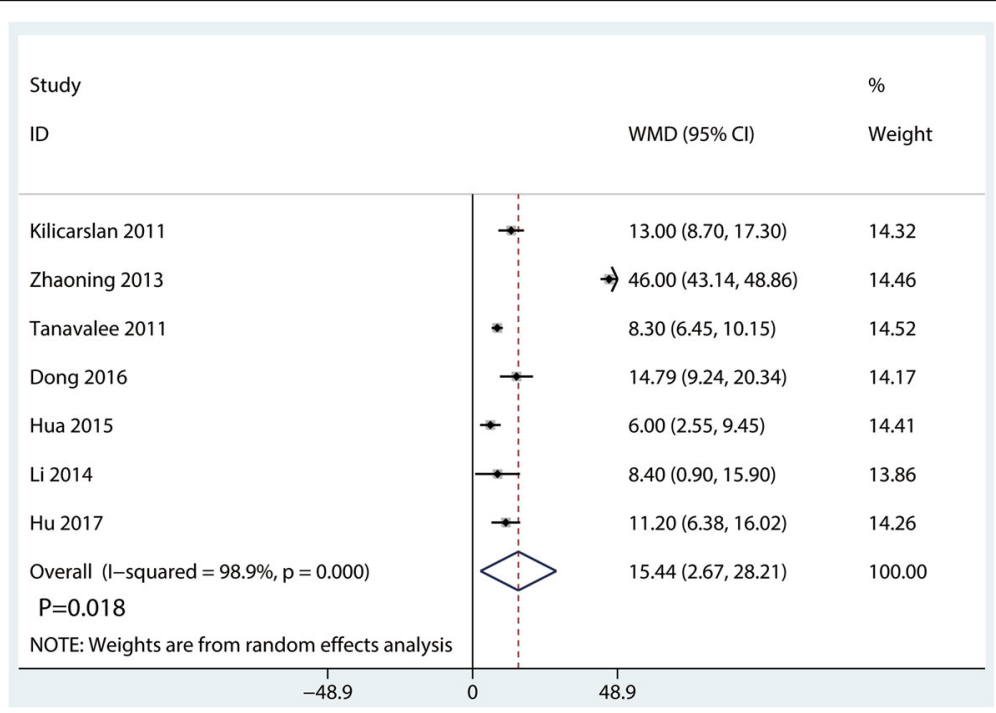

Fig. 9 Forest plots of the included studies comparing the operating time 


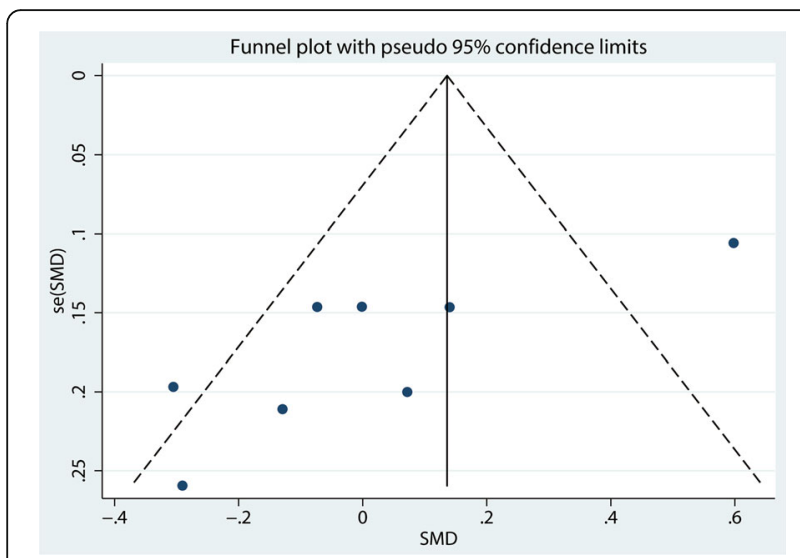

Fig. 10 Funnel plot of the pain scores

that synovectomy will cause more blood loss and operating time. And there was no significant difference between the pain scores, range of motion, and postoperative KSS.

A major strength of the current meta-analysis was that we included 10 RCTs and increased the credibility of the meta-analysis. Kooner et al. [8] performed a meta-analysis and found that there is currently no evidence to support the use of synovectomy in TKA for primary OA, as it provides no clinical benefit and increases postoperative blood loss. However, in that meta-analysis, only three RCTs were included and the author concluded in his meta-analysis that the major limitation of this review was the lack of studies included for analysis, as well as a lack of raw data. In the current meta-analysis, we included $10 \mathrm{RCTs}$ and thus provided relative confidence level.

Fernandez-Madrid et al. [20] suggest that synovitis occurs in OA or RA patients and might be a contributing cause of pain, and it has been proposed that surgical synovectomy can reduce pain and improve function after TKA.
Most evidence for synovectomy stems from much of the literature addressing TKA in inflammatory arthropathies and other inflammatory conditions [21, 22]. Most studies have reported favorable results following synovectomy combined with other specific surgical procedures [23]. Inflammation in OA is unlikely the cause of persistent pain postoperatively. The potential reason was patellar maltracking, improper placement of the prosthesis, and infection.

Functional and clinical KSSs were used to assess the postoperative knee function. However, we did not find any benefit of synovectomy for patients prepared for TKA. Zhaoning et al. [5] reported that postoperative recovery was not affected by retention or excision of the synovial membrane of the knee joint. Krackow [6] recommended that as little synovium as possible should be removed, and Yasgur et al. [24] recommended that only sufficient synovium should be excised to ensure adequate visualization. In current clinical practice, postoperative rehabilitation exercise was the main factor that affected the functional and clinical KSS. Since the postoperative rehabilitation was similar, thus no significant difference was observed in these two groups.

As for total blood loss and operating time, we found that synovectomy will increase the total blood loss and the operating time. Kooner et al. [8] also found that postoperative blood loss was significantly lower in synovium-retaining TKA group and the difference was statistically significant $(\mathrm{MD}=99.41 ; 95 \% \mathrm{CI}, 45.08-153.75)$. Pooled results in this meta-analysis indicated that the synovectomy group was associated with an increase of the total blood loss (WMD = 116.71, 95\% CI 78.63-154.79, $P=0.000$ ).

There were several limitations in this meta-analysis: (1) only 10 RCTs were included, which might have affected the precision of the effect size estimations; (2) follow-up in the included studies ranged from $24 \mathrm{~h}$ to

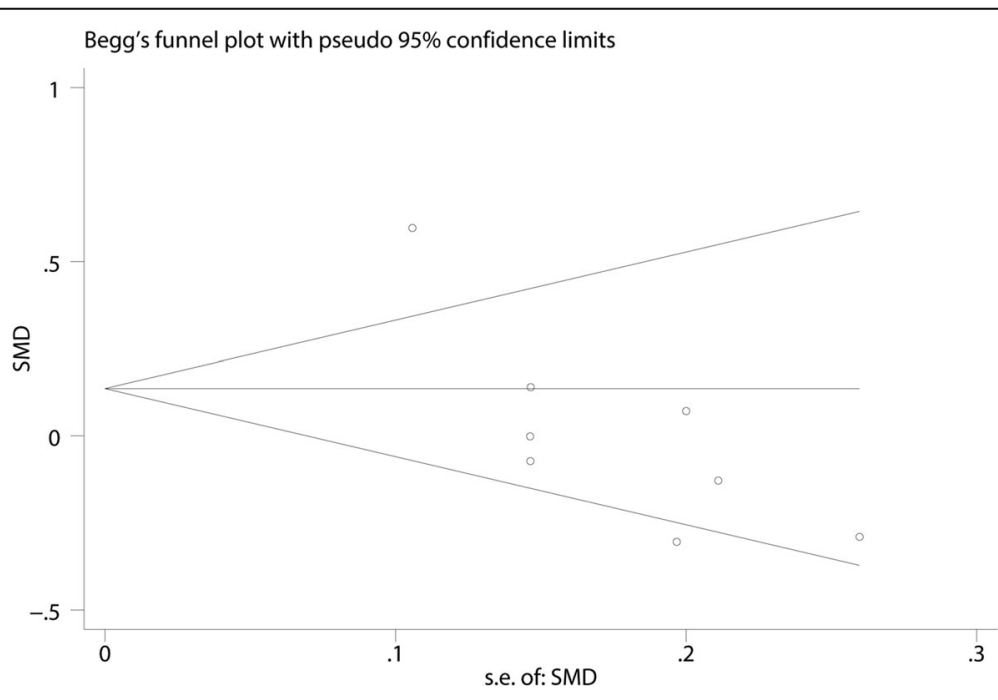

Fig. 11 Begg's test of the pain scores 


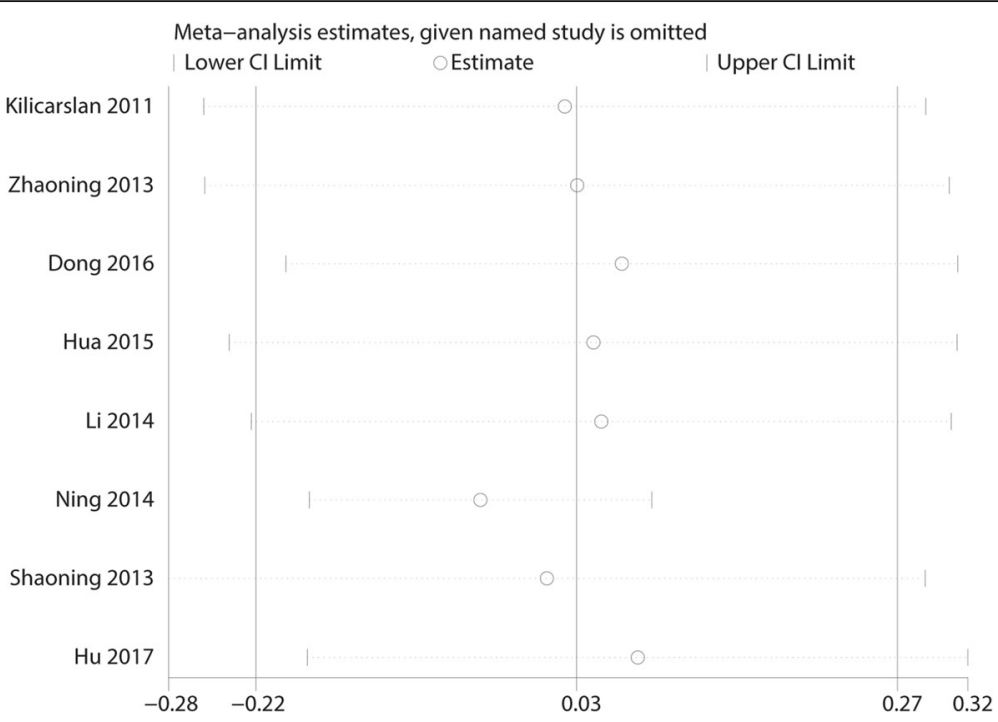

Fig. 12 Sensitivity analysis of the pain scores

6 month, and the relatively short-term follow-up may underestimate the complication rate; (3) perioperative nursing may be different and thus may cause the heterogeneity; (4) the follow-up duration in the included studies were relatively short and long-term follow-up was needed; and (5) publication bias existed in the pain scores and may affect the final results.

\section{Conclusion}

In conclusion, current evidence indicates that synovectomy has no effects on the final clinical outcomes for OA patients undergoing TKA. It will increase the total blood loss and the operating time during TKA. Because the sample size and the number of included studies were limited, a multi-center RCT is needed to identify the effects of synovectomy in reducing pain after TKA.

\section{Abbreviations}

Cl: Confidence interval; KSS: Knee Society Score; OA: Osteoarthritis; PRISMA: Preferred Reporting Items for Systematic Reviews and Meta-Analyses; RA: Rheumatoid arthritis; RCTs: Randomized controlled trials; SMD: Standard mean difference; TKA: Total knee arthroplasty; WMD: Weighted mean difference

\section{Acknowledgements}

None

\section{Funding}

There is no funding for this article.

\section{Availability of data and materials}

We state that the data will not be shared because all the raw data are present in the figures included in the article.

\section{Authors' contributions}

ZQZ and JX conceived the study design. RLW and ZQZ performed the study, collected the data, and contributed to the study design. LNX and RLW prepared the manuscript. ZQZ and JX edited the manuscript. All authors read and approved the final manuscript.
Ethics approval and consent to participate

None

Consent for publication

Not applicable

Competing interests

The authors declare that they have no competing interests.

\section{Publisher's Note}

Springer Nature remains neutral with regard to jurisdictional claims in published maps and institutional affiliations.

Received: 19 January 2018 Accepted: 27 February 2018

Published online: 12 March 2018

\section{References}

1. Krasnokutsky S, Belitskaya-Levy I, Bencardino J, et al. Quantitative magnetic resonance imaging evidence of synovial proliferation is associated with radiographic severity of knee osteoarthritis. Arthritis Rheum. 2011:63:2983-91.

2. Momohara S, Ikeda M, Uchida K, Kawamura K, Mizumura T, Tomatsu T. Follow-up results of arthroscopic synovectomy for the rheumatoid knee. Mod Rheumatol. 2001;11:205-9.

3. Furuzawa-Carballeda J, Macip-Rodriguez PM, Cabral AR. Osteoarthritis and rheumatoid arthritis pannus have similar qualitative metabolic characteristics and pro-inflammatory cytokine response. Clin Exp Rheumatol. 2008;26:554-60.

4. Malkoc M, Korkmaz O. Results of arthroscopic synovectomy for treatment of synovial lipomatosis (lipoma arborescens) of the knee. J Knee Surg. 2017. doi: https://doi.org/10.1055/s-0037-1604440. [Epub ahead of print]

5. Zhaoning $X, X u Y$, Shaoqi T, Baiqiang H, Kang S. The effect of synovectomy on bleeding and clinical outcomes for total knee replacement. Bone Joint J. 2013;95-b:1197-200

6. Krackow KA. The technique of total knee arthroplasty: C.V. England: Mosby Co.; 1990.

7. Gonzalez MH, Mekhail AO. The failed total knee arthroplasty: evaluation and etiology. J Am Acad Orthop Surg. 2004;12:436-46.

8. Kooner SS, Clark M. The effect of synovectomy in total knee arthroplasty for primary osteoarthritis: a meta-analysis. J Knee Surg. 2017:30:289-96.

9. Landis JR, Koch GG. An application of hierarchical kappa-type statistics in the assessment of majority agreement among multiple observers. Biometrics. 1977:33:363-74.

10. Landis JR, Koch GG. The measurement of observer agreement for categorical data. Biometrics. 1977;33:159-74. 
11. Dong N, Ke M, Huang L. The effects of synovectomy for blood loss and clinical outcomes for total knee arthroplasty. The Journal Of Medical Theory And Practice. 2016:44:108-10.

12. Hua Z, Zhang J, Liu G, Han W, Zhao W, Pan W. Influeding of synovectomy for total knee replacement bleeding and clinical effect. Heilongjiang Medicine Journal. 2015;26:749-52.

13. Kilicarslan $\mathrm{K}$, Yalcin $\mathrm{N}$, Cicek $\mathrm{H}$, et al. The effect of total synovectomy in total knee arthroplasty: a prospective randomized controlled study. Knee Surg Sports Traumatol Arthrosc. 2011;19:932-5.

14. Z-g L, Yang X-m, Yang H-j, Feng $H$. The clinical effect of synovectomy for primary osteoarthritis: a randomized controlled trial. Laboratory Medicine and Clinic. 2014;105:636-7.

15. Ning J, Tian S-q, Yang X, Zhao X, Sun K. The efficacy of synovectomy for osteoarthritis patients. Qilu Medical Journal. 2014;35:519-21.

16. Shaoning $X, X u Y$, Shaogi $T$, Baigiang $H$, Kang $S$. Effect of synovectomy on bleeding and clinical outcomes for total knee replacement. Chinese Journal of Clinicians (Electronic Edition). 2013;36:111-4.

17. Tanavalee A, Honsawek S, Rojpornpradit T, Sakdinakiattikoon M, Ngarmukos S. Inflammation related to synovectomy during total knee replacement in patients with primary osteoarthritis: a prospective, randomised study. J Bone Joint Surg Br. 2011;93:1065-70.

18. Yang B, Ning J, Tian S, Kang S. Effect of synovectomy on clinical outcome of bilateral total knee arthroplasty for osteoarthritis patients. Qingdaodaxuexuebao. 2016:43:40-2.

19. Hu H, Li Y, Li Z, Sun P. Effect of synovectomy on clinical outcome of total knee arthroplasty for osteoarthritis. Chinese Journal of Bone and Joint Injury. 2017;32:365-7.

20. Fernandez-Madrid F, Karvonen RL, Teitge RA, Miller PR, An T, Negendank WG. Synovial thickening detected by MR imaging in osteoarthritis of the knee confirmed by biopsy as synovitis. Magn Reson Imaging. 1995;13:177-83.

21. Kubacki J, Kokosz M, Grygorowicz M, Adamczyk-Bujniewicz H. The value of synovectomy of the knee in the treatment of rheumatoid arthritis. Ortop Traumatol Rehabil. 2006;8:99-105.

22. Tanaka N, Sakahashi H, Sato E, Hirose K, Isima T. Influence of the infrapatellar fat pad resection in a synovectomy during total knee arthroplasty in patients with rheumatoid arthritis. J Arthroplast. 2003;18:897-902.

23. Matsui $\mathrm{N}$, Moriya $\mathrm{H}$, Kitahara $\mathrm{H}$. The use of arthroscopy for follow-up in knee joint surgery. Orthop Clin North Am. 1979;10:697-708.

24. Yasgur DJ, Scuderi GR, Insall JN. Medial release for fixed-varus deformity. 2006.

\section{Submit your next manuscript to BioMed Central and we will help you at every step:}

- We accept pre-submission inquiries

- Our selector tool helps you to find the most relevant journal

- We provide round the clock customer support

- Convenient online submission

- Thorough peer review

- Inclusion in PubMed and all major indexing services

- Maximum visibility for your research

Submit your manuscript at www.biomedcentral.com/submit

) Biomed Central 